Ingeniería

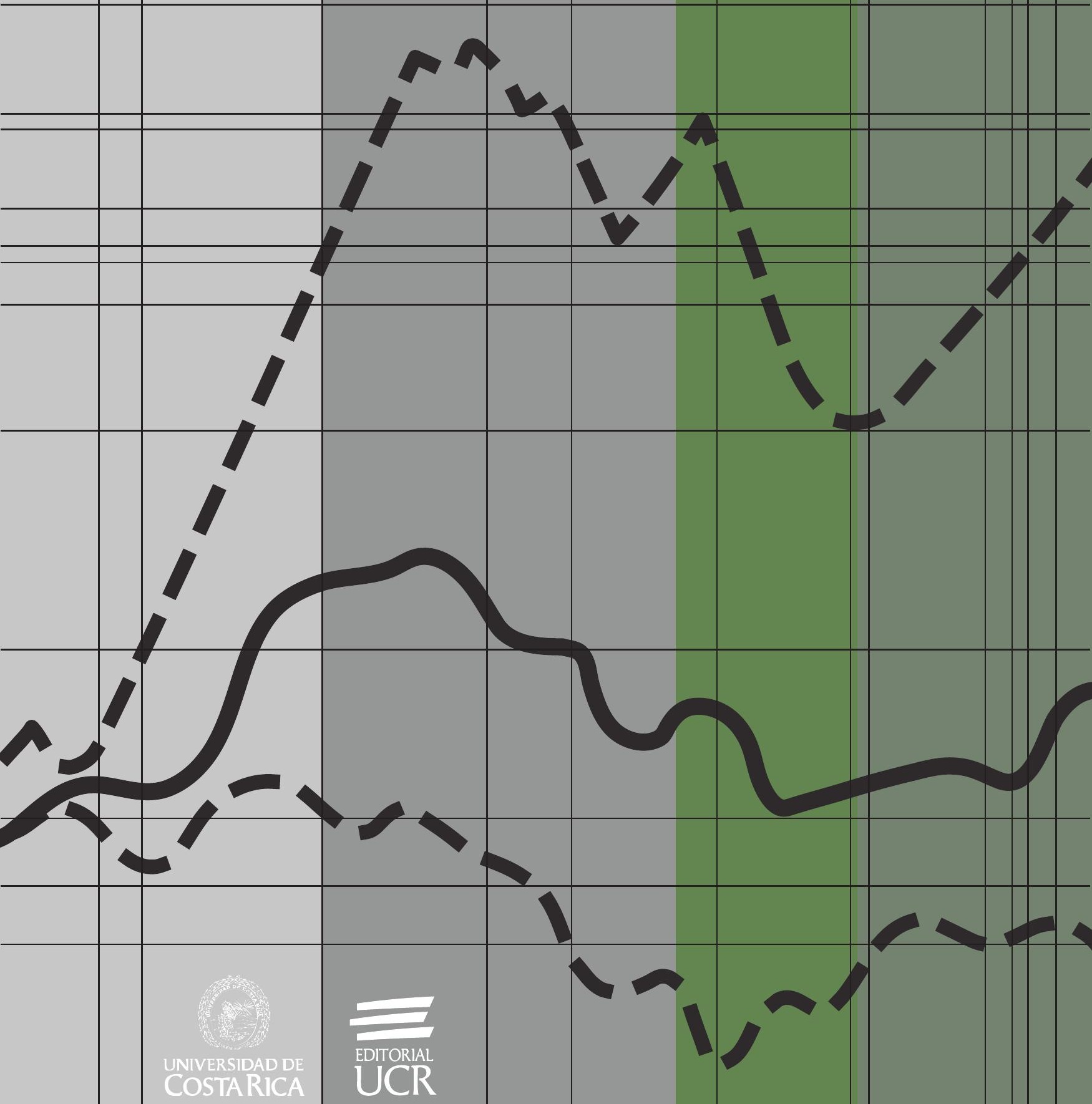




\title{
Determinación de las propiedades mecánicas del cuero fino de vaca sometido a condiciones variadas de temperatura y humedad
}

\section{Determination of the Mechanical Properties of the Fine Leather Subjected to Varied Temperature and Humidity Conditions}

\author{
José Miguel Briguido \\ Universidad Nacional de La Rioja. Argentina. \\ m.briguido@larioja.gov.a \\ Julieta Fabiana Calderón \\ Universidad Nacional de La Rioja. Argentina. \\ julicalderon511@yahoo.com.ar \\ Liliana Laura Recchioni \\ Universidad Nacional de La Rioja. Argentina. \\ liliana.recchioni@gmail.com \\ Adriana Cecilia Díaz \\ Universidad Nacional de La Rioja. Argentina. \\ acd_1985_10@hotmail.com
}

\section{Resumen}

Dadas evidencias circunstanciales de que la resistencia del cuero varía en diferentes niveles de temperatura y humedad, se someten distintas probetas de cuero fino de vaca de alta calidad y costo utilizado para la industria del calzado a ensayos mecánicos bajo diferentes condiciones de esos factores. Para ello se mide: resistencia a la tracción o tensión, alargamiento porcentual por una carga, alargamiento a la rotura y fuerza de desgarramiento.

El objetivo es observar si esas variables del cuero sufren alguna modificación significativa cuando se los somete a determinadas condiciones de temperatura y humedad. Se utilizó un modelo completamente aleatorizado y un modelo estadístico con covariable utilizando estimaciones de parámetros REML (Restricted Maximum Likelihood) y pruebas a posteriori.

Se concluye que existe gran heterogeneidad en las mediciones en todas las variables bajo diferentes tratamientos de humedad y temperatura, dados los altos valores de coeficientes de variación y que las propiedades mecánicas del tipo de cuero no tienen variación significativa cuando se somete a condiciones diferentes de lo normal. 
Palabras clave:

Cuero, materiales, resistencia, temperatura, humedad.

\begin{abstract}
IThis project consists of subjecting different types of leather (SOFT) of higher quality and cost, to three types of mechanical tests: determination of tensile strength and percentage elongation, determination of tear strength by the method of 2 edges and tear test at a sewing point by the two-hole method.

The objective is to observe if the resistance of the leather to certain conditions of temperature and humidity undergoes some significant modification. We used a completely randomized model and a covariate statistical model and a posteriori tests were performed according to Fisher.

The parameter estimation was performed according to the Restricted Maximum Likelihood (REML) method using the InfoStat software. After testing for equal treatment effects, normality is tested with Shapiro Wilks at a significance level of $1 \%$.

It is concluded that there is great heterogeneity in the measurements in all the variables under different treatments of humidity and temperature, given the high values of coefficients of variation, and that the mechanical properties of the type of soft leather do not have significant variation when subjected to conditions different than usual.
\end{abstract}

Key words:

Industry, testing, materials, breakages, standards. 


\section{INTRODUCCIÓN}

El cuero tiene propiedades únicas como material. Es transpirable, permeable al vapor de agua, almacena la humedad, no se desgarra, es elástico y dispone de una capacidad de adaptación. A causa de estas propiedades, el cuero tiene ciertos requerimientos, sobre todo en el ámbito del calzado, para mantener su funcionalidad.

Los cueros son muy absorbentes si en el curtido no se ha tenido en cuenta la posibilidad de rechazar el agua, y tienen que ser protegidos de la humedad cuando el proceso de curtido no sigue las normas establecidas para tal fin. Por las influencias del medio ambiente, se reduce claramente el contenido original en ceras y grasas en el cuero, que son necesarias para la flexibilidad y elasticidad.

Existen antecedentes sobre el análisis de la suavidad y flexibilidad del cuero sometido a diferentes condiciones de curtido y evaluadas sobre condiciones iniciales del cuero tales como su contenido de agua o su espesor, propuestas en la tesis doctoral de Wenge (1999).

Boccone, Fontana y Kamp (1977a) analizaron la distribución de las cargas de rotura del cuero sobre distintas superficies o ubicaciones analizando las propiedades del cuero y recomendando las zonas que deben evitarse para el corte destinado a fabricación del calzado. Asimismo Boccone, Fontana y Kamp (1977b) analizaron la relación entre las cargas de rotura por tracción medidas a través de dos instrumentos diferentes con el fin de proporcionar información que facilite las mediciones de las propiedades del cuero.

Existen antecedentes de Boccone y Fontana (1979a) sobre el estudio de propiedades del cuero que inciden sobre el desgarro, pero sometidas a diferentes procesos en el curtido especialmente relacionado al agregado de aceite de pezcado, el recurtido y condiciones de secado.

Por otra parte, también Boccone y Fontana (1979b) también analizaron la incorporación de substancias químicas y procesos alternativos para la mejora de la resistencia de los cueros logrando resultados superadores, aunque siempre focalizado en el proceso de curtido.

Silva Trejos (2005) ha realizado estudios sobre calzado de seguridad disponibles en el mercado costarrincense relacionados a algunas propiedades físicas y químicas de cuero y la correlación entre algunas de ellas, sin analizar el sometimiento a diferentes condiciones.

De acuerdo a la información disponible se verifica que es necesario conocer el comportamiento del cuero destinado a la fabricación de calzado, prendas y productos de tapicería, cuando es sometido a condiciones de temperatura y humedad distintas a las normales.

Por consiguiente, se deben verificar las propiedades del cuero y conservación debido a que el objetivo del cuidado del cuero es mantener las propiedades típicas y al mismo tiempo darle una excelente apariencia.

Las curtiembres y los compradores de cuero han constatado que el método de los dos orificios es indicativo de la resistencia del cuero al desgarro. Es un valor particular 
para estimar la resistencia del cuero al esfuerzo de desgarramiento en las costuras, sobre todo para la fabricación de calzado, prendas y productos de tapicería.

A la fecha no se cuenta con antecedentes de estudios referidos a diferentes condiciones ambientales en la provincia de La Rioja; y al tener la región temperaturas elevadas durante gran parte del año, se considera que la contribución al avance del conocimiento científico es de gran importancia.

Actualmente en la provincia La Rioja se encuentran radicadas importantes empresas industriales destinadas al procesamiento del cuero y a la elaboración de distintos productos de este (calzado, tapicería, indumentaria, etc.), por lo que resulta de suma importancia el estudio de las propiedades más significativas que tiene dicho material y la influencia que puede producir la variación de determinados parámetros termodinámicos, como son la temperatura y la humedad.

El objetivo general del presente trabajo es determinar las propiedades mecánicas del cuero fino denominado también badana o tipo SOFT, en las diferentes condiciones de temperatura y humedad a las cuales es sometido.

\section{MATERIALES Y MÉTODOS}

\subsection{Materiales}

La máquina universal de ensayos utilizada para realizar los diferentes ensayos posee las características mencionadas en la figura 1.

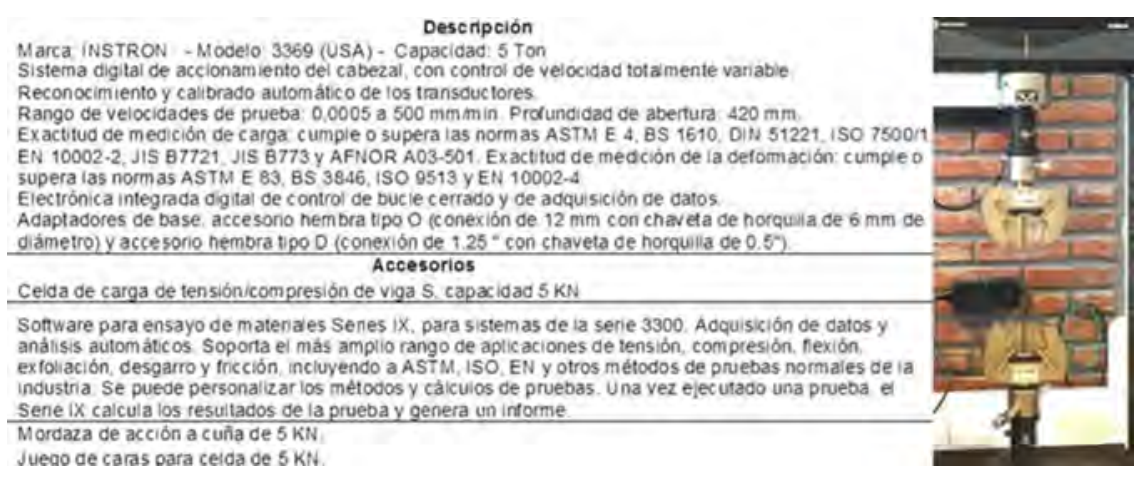

Figura 1. Características de la máquina universal utilizada para las pruebas.

Se utilizarán como unidades experimentales 3 tipos de probeta de cuero: Probeta A para la determinación de la resistencia a la tracción, del alargamiento porcentual y del alargamiento a la rotura; Probeta B para la determinación de la fuerza al desgarramiento en un punto de costura mediante el método de los 2 orificios; y Probeta $\mathrm{C}$ para la determinación de la fuerza al desgarramiento mediante el método de los 2 bordes. Las probetas se presentan en la figura 2 . 
A

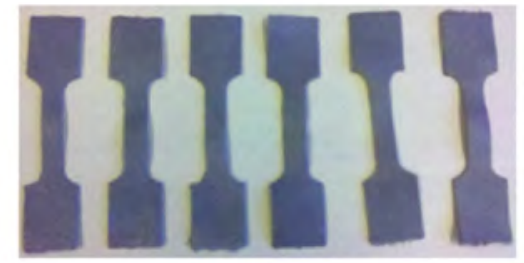

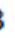
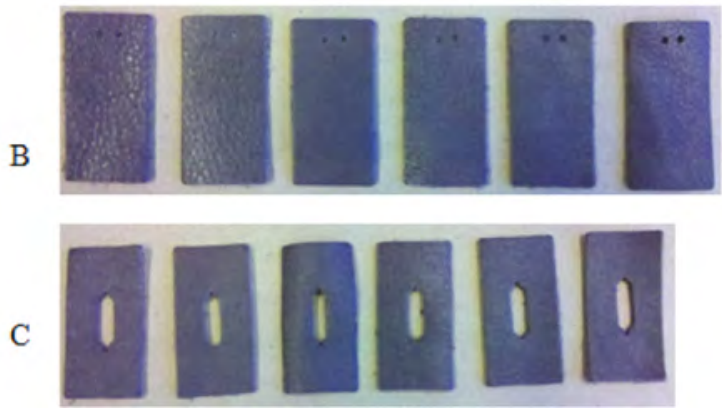

Figura 2. Probetas A, B y C.

Para la preparación y acondicionamiento de las probetas, se utilizó la Norma IRAM 8555 (2005), la cual establece el procedimiento para la preparación de las probetas de cuero para los ensayos físicos y mecánicos y la atmosfera normalizada para el acondicionamiento y ensayo. De acuerdo a esta norma, se trabajó con una atmósfera normalizada de Temperatura: $23{ }^{\circ} \mathrm{C} \pm 2$ y Humedad Relativa: $50 \% \pm 5$.

Los sacabocados fueron confeccionados por el área de mantenimiento de la misma empresa proveedora de los cueros, de acuerdo a las normas establecidas IRAM 8511 (2006), IRAM 8513 (2006) e IRAM 8514 (2006) (ver figura 3).

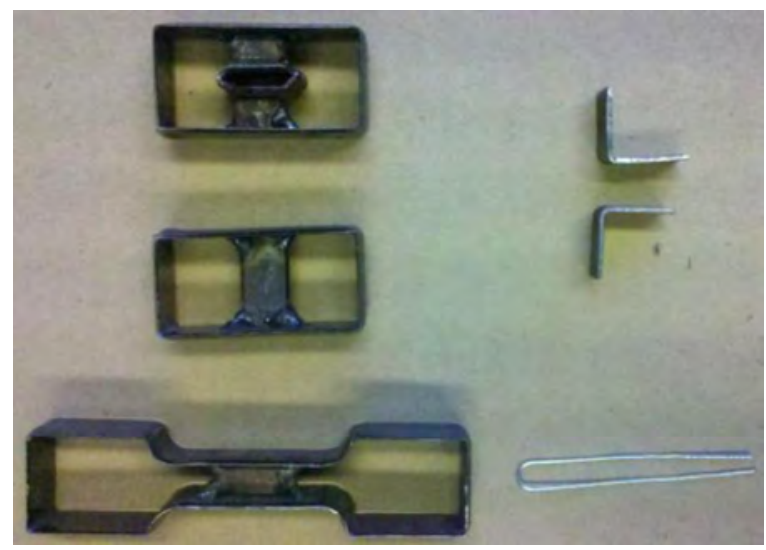

Figura 3. Sacabocados.

La confección de probetas se realizó con un muestreo del cuero provisto por la empresa, cumpliendo con lo indicado en la norma IRAM 8522 (2005) (ver figura 4). 

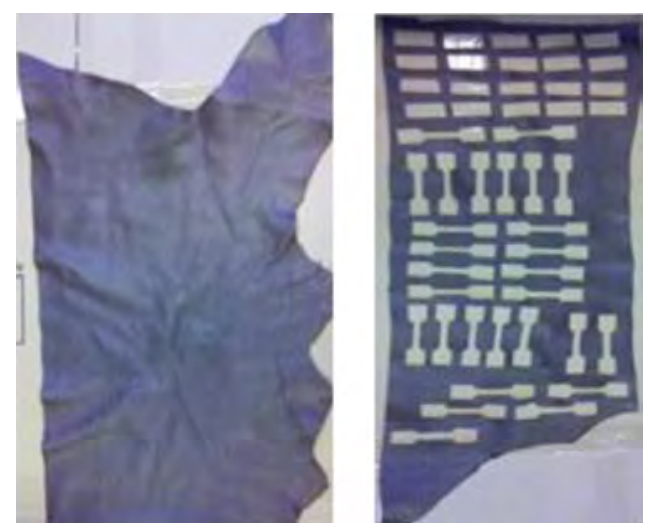

Figura 4. Muestra de cuero fino de vaca utilizado para la confección de las probetas.

Se preparan las probetas aplicando el sacabocado en la superficie de la flor. Estas fueron cortadas a través de las maquina universal, utilizando compresión (figura 5) para lograr un corte rápido y parejo.

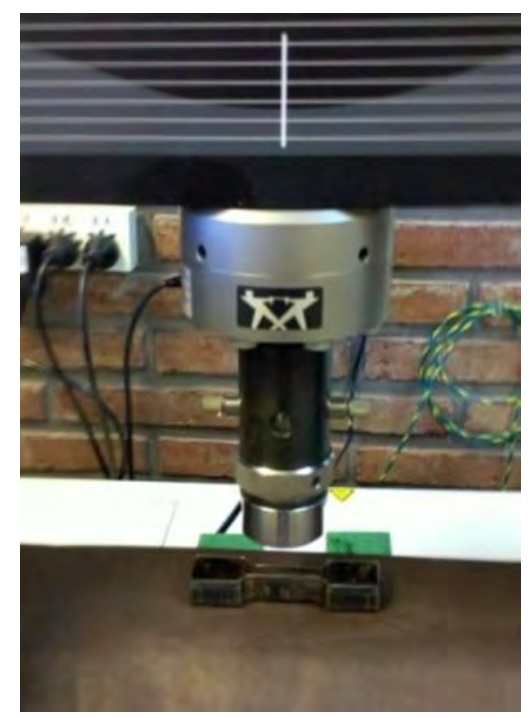

Figura 5. Preparación de probetas para el corte.

\subsection{Tratamientos}

Para la ejecución de los distintos ensayos se consideraron distintos niveles de los factores temperatura y humedad en forma independiente, controlando en todos los casos la temperatura ambiente a $25^{\circ}$ y humedad ambiente a $50 \%$.

Para evaluar el factor humedad, se realizó el ensayo correspondiente a la absorción de agua, para el que se utilizaron 30 probetas de cada tipo A, B y C. Se adjudicaron 6 
unidades experimentales (por cada tipo de probeta) aleatoria e independientemente a los siguientes tratamientos: 0 horas (control), 1 hora, 6 horas, 12 horas y 24 horas. Estos tratamientos corresponden a la cantidad de horas que el cuero estará sumergido en agua.

Para el caso de Temperatura también se seleccionaron 30 probetas de cada tipo A, B y C, distribuyendo aleatoriamente 6 unidades a cada uno de los 5 niveles del factor temperatura: $20^{\circ} \mathrm{C}$ (Control), $40^{\circ} \mathrm{C}, 60^{\circ} \mathrm{C}, 80^{\circ} \mathrm{C} \mathrm{y} 100^{\circ} \mathrm{C}$.

\subsection{Variables a observar}

En las probetas A se midieron las variables resistencia a la tracción, alargamiento a una carga determinada y alargamiento a la rotura una vez culminado el proceso que determine el tratamiento de humedad y de temperatura. Además, se mide la variable área antes de someterlas al tratamiento, con la finalidad de utilizarla en el modelo como covariable, lo cual controlará las características de la unidad experimental para evitar que condicione el resultado del experimento. Se las definen de la siguiente manera:

Resistencia a la Tracción o Tensión o TR [1] Siendo F la Fuerza Máxima registrada, en Newton (N) y A el Área de la probeta (en mm2). Para ello la TR se mide en MPa.

$$
T_{R}=\frac{F}{A}
$$

El Área A [2] es el producto entre el ancho medio y el espesor medio de la probeta, ambos en $\mathrm{mm}$.

$$
A=w \cdot t
$$

Alargamiento porcentual Ei [3] producido por una carga determinada $(50 \mathrm{~N})$, siendo Li la separación entre las mordazas bajo una carga determinada y LO la separación inicial entre mordazas.

$$
E_{i}=\frac{L_{i}-L_{0}}{L_{0}} 100
$$

Alargamiento a la rotura Eb [4], siendo L2 la separación entre las mordazas a la rotura y L0 la separación inicial entre mordazas

$$
E_{b}=\frac{L_{2}-L_{0}}{L_{0}} 100
$$


Para los ensayos de tratamientos de Humedad y Temperatura en las probetas B y C se observará como variable respuesta a la Fuerza (Fmx) y como covariable al Espesor Promedio Inicial (EP). La Fuerza es la Fuerza de Desgarramiento en newton (N) y el Espesor Promedio de las Muestras medido en milímetros $(\mathrm{mm})$ de acuerdo a la Norma IRAM 8509 (2006).

\subsection{Métodos}

Tanto el instrumental, las probetas de ensayo y demás elementos intervinientes en el ensayo se ajustaron a las normas vigentes.

El ensayo de resistencia a la tracción y del alargamiento porcentual consiste en estirar una probeta a una velocidad especificada hasta que la dicha probeta se rompa.

El ensayo al desgarramiento en un punto de costura por el método de los 2 orificios consiste en considerar una probeta de cuero con dos orificios en uno de los extremos. A través de dichos orificios se pasa un alambre de acero, doblado en forma de U que se sujeta a un par de mordazas de la máquina de ensayo, sujetando al extremo libre de la probeta al otro par de mordazas. Se aplica una tensión creciente y se determina el valor de la carga en el momento en que se produce el desgarramiento de la probeta.

La norma última mencionada establece la forma y cantidad de probetas a ensayar, las condiciones del ensayo y los valores que deben determinarse.

En el ensayo al desgarramiento en un punto de costura por el método de los 2 orificios se tomarán probetas de cuero, con dos orificios circulares en uno de los extremos. A través de dichos orificios se pasa un alambre de acero, doblado en forma de U que se sujeta a un par de mordazas de la máquina de ensayo, sujetando al extremo libre de la probeta al otro par de mordazas. Se aplica una tensión creciente y se determina el valor de la carga en el momento en que se produce el desgarramiento de la probeta.

El ensayo de resistencia al desgarramiento mediante el método de los 2 bordes consiste en colocar una probeta que tiene un orificio de una forma específica sobre los extremos levantados de un par de portaprobetas unidas a las mordazas de la máquina de ensayo de tracción. Se registra la mayor fuerza ejercida durante el desgarramiento de la probeta.

\subsection{Análisis estadístico}

Se analizó de manera descriptiva la información proveniente de las variables. Se utilizó el modelo estadístico [5] con diseño completamente aleatorizado a un factor y con covariable.

$$
y_{i j}=\mu+\tau_{i}+\beta x_{i j}+\xi_{i j}
$$

Donde $\mathrm{y}_{\mathrm{ij}}$ es la jotaésima observación de la variable respuesta del iésimo nivel de temperatura o humedad. 
$\tau_{i}$ es el efecto del iésimo nivel de tratamiento, $\beta$ es el coeficiente de la covariable y $\xi_{i j}$ es el error aleatorio distribuido $\mathrm{N}(0 ; \mathrm{R} \sigma 2)$.

La estimación de parámetros se realizó según el método REML(Máxima verosimilitud restringida). Luego de las pruebas de igualdad de efectos de tratamientos se realizan pruebas a posteriori. El supuesto de normalidad que se establece en el modelo estadístico sobre el error aleatorio se controló mediante el test de Shapiro Wilks con nivel de significación del 1\%.

\section{RESULTADOS}

\subsection{Humedad}

\subsubsection{Probeta A}

En la tabla 1 se observan los valores que describen el comportamiento de la tensión, alargamiento porcentual a $50 \mathrm{~N}$ (alar50) y alargamiento a la rotura (alarot) correspondiente a los diferentes tratamientos.

Tabla 1. Valores descriptivos en las pruebas de humedad para Probeta A.

\begin{tabular}{cccccccc}
\hline Tratamiento & Variable & $\mathrm{n}$ & Media & D.E. & CV & Mín & Máx \\
\hline Control & tensión & 6 & 11.27 & 2.36 & 20.97 & 8.18 & 14.50 \\
$1 \mathrm{hr}$ & tensión & 6 & 15.94 & 2.60 & 16.29 & 11.82 & 18.76 \\
$6 \mathrm{hrs}$ & tensión & 6 & 16.34 & 2.92 & 17.86 & 12.36 & 21.14 \\
$12 \mathrm{hrs}$ & tensión & 6 & 15.60 & 1.89 & 12.14 & 12.86 & 18.64 \\
$24 \mathrm{hrs}$ & tensión & 6 & 15.38 & 1.62 & 10.55 & 13.05 & 17.36 \\
Control & alar50 & 6 & 37.92 & 13.49 & 35.58 & 17.63 & 50.22 \\
$1 \mathrm{hr}$ & alar50 & 6 & 41.14 & 18.76 & 45.60 & 20.91 & 68.74 \\
$6 \mathrm{hrs}$ & alar50 & 6 & 31.49 & 12.41 & 39.40 & 15.97 & 46.04 \\
$12 \mathrm{hrs}$ & alar50 & 6 & 30.96 & 9.70 & 31.34 & 22.65 & 49.55 \\
$24 \mathrm{hrs}$ & alar50 & 6 & 33.76 & 10.34 & 30.62 & 24.25 & 48.49 \\
Control & alarot & 6 & 74.90 & 17.99 & 24.01 & 51.09 & 91.88 \\
$1 \mathrm{hr}$ & alarot & 6 & 79.95 & 24.11 & 30.16 & 56.27 & 119.11 \\
$6 \mathrm{hrs}$ & alarot & 6 & 72.04 & 19.94 & 27.68 & 48.44 & 103.64 \\
$12 \mathrm{hrs}$ & alarot & 6 & 70.79 & 17.80 & 25.14 & 56.31 & 103.43 \\
$24 \mathrm{hrs}$ & alarot & 6 & 71.91 & 14.09 & 19.60 & 57.47 & 88.78 \\
\hline
\end{tabular}

En el caso de la tensión sometida al tratamiento control, presenta el menor valor promedio. Estos promedios son superiores a partir de la primera hora de exposición.

El estudio de las diferencias de medias utilizando el modelo de covariancia para las variables observadas dio por resultado que en la variable tensión ( $\mathrm{p}=0.0075$ ) hay un efecto 
significativo de los tratamientos, el tratamiento inicial (control) es el que presenta un promedio menor y las demás medias no difieren entre sí. En las características de alargue porcentual $(\mathrm{p}=0.7156)$ y alargue a la rotura $(\mathrm{p}=0.9506)$ no existe efecto de la humedad.

Mediante el análisis gráfico de residuales y la prueba Shapiro Wilks se controló la no violación de supuestos (tensión $\mathrm{p}=0.2386$, alar50 p= 0.1260 y alarot $\mathrm{p}=0.0381$ ).

\subsubsection{Probeta B}

Los análisis para la probeta B se realizan mediante la variable respuesta Fuerza (Fmx), cuyos valores descriptivos según tratamientos se presentan en la tabla 2.

Se detectaron diferencias significativas entre tratamientos $(p=0.0013)$ a las $24 \mathrm{hs}$ la fuerza disminuye, y fue adecuada la inclusión de la covariable $(\mathrm{p}<0.0001)$, por lo tanto en términos medios el área de las probetas difieren según los tratamientos. No se rechaza la prueba de normalidad $(\mathrm{p}=0.0220)$.

Tabla 2. Valores descriptivos en las pruebas de humedad para Probeta B.

\begin{tabular}{cccccccc}
\hline Trata & Variable & $\mathrm{n}$ & Media & D.E. & CV & Mín & Máx \\
\hline Inicial & Fmx & 6 & 113.80 & 38.73 & 34.03 & 67.00 & 163.66 \\
$1 \mathrm{hr}$ & Fmx & 6 & 98.65 & 45.84 & 46.46 & 54.55 & 158.42 \\
$6 \mathrm{hr}$ & Fmx & 6 & 142.22 & 25.75 & 18.10 & 101.17 & 177.12 \\
$12 \mathrm{hr}$ & Fmx & 6 & 132.46 & 66.78 & 50.42 & 60.40 & 224.49 \\
$24 \mathrm{hr}$ & Fmx & 6 & 90.37 & 41.88 & 46.35 & 64.52 & 174.40 \\
\hline
\end{tabular}

\subsubsection{Probeta C}

En la probeta $\mathrm{C}$ se midió la variable Fuerza presentando los valores de la respuesta a los tratamientos que se describen en la tabla 3.

Tabla 3. Valores descriptivos en las pruebas de humedad para Probeta C.

\begin{tabular}{cccccccc}
\hline Trata & Variable & $\mathrm{n}$ & Media & D.E. & CV & Mín & Máx \\
\hline $\begin{array}{c}\text { Ini- } \\
\text { cial }\end{array}$ & Fmx & 6 & 93.03 & 37.00 & 39.77 & 55.07 & 159.16 \\
$1 \mathrm{hr}$ & Fmx & 6 & 73.19 & 13.13 & 17.94 & 52.92 & 86.47 \\
$6 \mathrm{hrs}$ & Fmx & 6 & 77.01 & 15.10 & 19.61 & 61.93 & 99.86 \\
$12 \mathrm{hrs}$ & Fmx & 6 & 56.47 & 9.07 & 16.06 & 48.43 & 71.36 \\
$24 \mathrm{hrs}$ & Fmx & 6 & 76.03 & 14.38 & 18.91 & 57.24 & 100.16 \\
\hline
\end{tabular}

Se detectaron diferencias significativas entre tratamientos $(\mathrm{p}=0.0140)$ y fue adecuada la inclusión de la covariable $(\mathrm{p}<0.0001)$. La fuerza es menor en el tratamiento de 12 hs.; 
las restantes medias no difieren. El supuesto de normalidad se mantiene según Shapiro Wilks $(\mathrm{p}=0.4079)$.

\subsection{Temperatura}

\subsubsection{Probeta A}

La aplicación de los tratamientos de temperatura en las probetas A, dieron por resultado los siguientes valores descriptivos para cada una de las 3 variables de respuestas observadas (tabla 4).

Tabla 4. Valores descriptivos en las pruebas de temperatura para Probeta A.

\begin{tabular}{cccccccc}
\hline trata & Variable & $\mathrm{n}$ & Media & D.E. & CV & Mín & Máx \\
\hline Inicial & tensión & 6 & 9.37 & 3.47 & 37.08 & 4.91 & 15.13 \\
40 & tensión & 6 & 9.63 & 1.39 & 14.46 & 7.66 & 11.81 \\
60 & tensión & 6 & 9.83 & 2.15 & 21.92 & 7.17 & 12.38 \\
80 & tensión & 6 & 8.80 & 1.62 & 18.39 & 7.21 & 11.79 \\
100 & tensión & 6 & 9.09 & 1.30 & 14.31 & 7.62 & 10.52 \\
Inicial & alar50 & 6 & 61.11 & 16.15 & 26.42 & 43.33 & 80.00 \\
40 & alar50 & 6 & 48.89 & 9.81 & 20.08 & 33.33 & 60.00 \\
60 & alar50 & 6 & 29.15 & 9.55 & 32.78 & 18.62 & 45.20 \\
80 & alar50 & 6 & 27.24 & 5.27 & 19.34 & 17.95 & 34.40 \\
100 & alar50 & 6 & 24.28 & 4.59 & 18.89 & 16.59 & 30.10 \\
Inicial & alrot & 6 & 67.14 & 15.65 & 23.31 & 48.03 & 83.56 \\
40 & alrot & 6 & 52.57 & 9.11 & 17.32 & 38.70 & 63.33 \\
60 & alrot & 6 & 59.95 & 11.62 & 19.39 & 43.33 & 72.58 \\
80 & alrot & 6 & 58.53 & 6.49 & 11.09 & 51.31 & 69.46 \\
100 & alrot & 6 & 51.71 & 9.93 & 19.21 & 36.89 & 64.50 \\
\hline
\end{tabular}

Dentro de cada tratamiento y para las tres variables se observó que la heterogeneidad es alta, dado que los coeficientes de variación son en mayoría mayor del $15 \%$.

No se detectaron diferencias significativas en Tensión ( $\mathrm{p}=0.8378)$ y en alargue a la rotura $(\mathrm{p}=0.1965)$, pero si en alargamiento a la carga $(\mathrm{p}<0.0001)$.

En alargamiento a la carga los mayores valores se obtienen en los tratamientos inicial y a $40^{\circ} \mathrm{C}$; en los restantes tratamientos no hay diferencias y sus valores medios son menores.

La covariable no es significativa en ninguna de las tres variables; por lo tanto, el área de las unidades experimentales es igual en términos medios, en los diferentes tratamientos $(\mathrm{p}=0.3685,0.3628,0.0844)$. 
Se cumple el supuesto de normalidad según Shapiro Wilks ( $\mathrm{p}=0.0219, \mathrm{p}=0.3269$, $\mathrm{p}=0.0267)$.

\subsubsection{Probeta B}

Los análisis para la probeta B se realizan mediante la variable respuesta Fuerza (Fmx), cuyos valores descriptivos según tratamientos se presentan en la tabla 5.

Tabla 5. Valores descriptivos en las pruebas de humedad para Probeta B.

\begin{tabular}{cccccccc}
\hline Trata & Variable & $\mathrm{n}$ & Media & D.E. & CV & Mín & Máx \\
\hline Inicial & Fmx & 6 & 135.23 & 36.87 & 27.26 & 89.14 & 192.43 \\
40 & Fmx & 6 & 109.41 & 16.86 & 15.41 & 89.01 & 130.05 \\
60 & Fmx & 6 & 114.92 & 23.50 & 20.45 & 85.63 & 145.82 \\
80 & Fmx & 6 & 126.87 & 32.15 & 25.34 & 81.61 & 165.67 \\
100 & Fmx & 6 & 108.49 & 20.22 & 18.63 & 83.33 & 133.74 \\
\hline
\end{tabular}

Si bien hay variaciones en los promedios, esta variable denota una gran variabilidad dentro de cada tratamiento. Por esta razón no se detecta una diferencia significativa entre tratamientos ( $\mathrm{p}=0.4592)$. Lo mismo ocurre con la covariable espesor (0.8504). Además, se cumple el supuesto de normalidad según Shapiro Wilks ( $\mathrm{p}=0.0179)$.

\subsubsection{Probeta C}

Si bien los valores promedio disminuyen a medida que la temperatura se incrementa (tabla 6), en esta probeta no se detectaron diferencias significativas en Fuerza $(\mathrm{p}=0.2316)$, y tampoco fue significativa la covariable $(\mathrm{p}=0.0891)$.

Tabla 6. Valores descriptivos en las pruebas de humedad para Probeta C.

\begin{tabular}{cccccccc}
\hline Trata & Variable & $\mathrm{n}$ & Media & D.E. & CV & Mín & Máx \\
\hline Inicial & Fmx & 6 & 109.07 & 9.97 & 9.15 & 97.18 & 125.22 \\
40 & Fmx & 6 & 100.20 & 14.04 & 14.02 & 79.82 & 113.51 \\
60 & Fmx & 6 & 95.16 & 34.66 & 36.42 & 45.30 & 150.97 \\
80 & Fmx & 6 & 88.95 & 26.82 & 30.15 & 53.11 & 133.34 \\
100 & Fmx & 6 & 75.93 & 13.72 & 18.08 & 48.93 & 85.76 \\
\hline
\end{tabular}

Se cumple el supuesto de normalidad según Shapiro Wilks ( $\mathrm{p}=0.4852)$. 
Para una mejor observación de los resultados obtenidos en todos los ensayos de propiedades, se presentan en la tabla 7.

Tabla 7. Síntesis de resultados.

\begin{tabular}{|c|c|c|c|}
\hline Tratamiento & Probeta & Variable & Resultado \\
\hline \multirow[t]{5}{*}{ Humedad } & \multirow[t]{3}{*}{ A } & Tensión & $\begin{array}{l}\text { En el tratamiento inicial se observa el menor valor. La tensión } \\
\text { en los demás tratamientos no difiere. }\end{array}$ \\
\hline & & Alargue porcentual & No hay diferencias \\
\hline & & Alargue a la rotura & No hay diferencias \\
\hline & B & Fuerza & $\begin{array}{l}\text { Se mantiene constante hasta las } 12 \text { hs. A las } 24 \text { hs. se observa } \\
\text { disminución }\end{array}$ \\
\hline & $\mathrm{C}$ & Fuerza & $\begin{array}{l}\text { No hay diferencias con excepción del tratamiento a las } 12 \mathrm{hs} \text {, } \\
\text { donde la fuerza es menor. Se recomienda repetir el ensayo de } \\
\text { las } 12 \mathrm{hs} \text {. }\end{array}$ \\
\hline \multirow[t]{5}{*}{ Temperatura } & \multirow[t]{3}{*}{ A } & Tensión & No hay diferencias \\
\hline & & Alargue porcentual & $\begin{array}{l}\text { En el tratamiento inicial y } 40^{\circ} \mathrm{C} \text { se presentan los mayores } \\
\text { valores, en los restantes no hay diferencias. Disminuye el } \\
\text { alargue con la temperatura. }\end{array}$ \\
\hline & & Alargue a la rotura & No hay diferencias \\
\hline & B & Fuerza & No hay diferencias \\
\hline & $\mathrm{C}$ & Fuerza & No hay diferencias \\
\hline
\end{tabular}

Luego cuando se expone al material a ensayos de humedad, hay variación en la tensión (Probeta A) y la fuerza (probetas B y C). En el caso de temperatura solo varía en el alargue (Probeta A).

\section{DISCUSIÓN Y CONCLUSIONES}

En general, existe una gran variabilidad o heterogeneidad en las mediciones de todas las variables observadas según los distintos tratamientos de humedad y temperatura. Esto implica que se trabaja con una baja precisión, tal como lo revelan los altos valores del coeficiente de variación. Por lo tanto, es necesario determinar en futuras experimentaciones qué factores influyen en este comportamiento, siendo uno de los probables las propiedades iniciales o zonas de extracción de las probetas.

Para el tratamiento humedad, la resistencia a la tracción o tensión presenta diferencias significativas. Es mayor en la exposición a la humedad, y en el alargue porcentual y el alargue a la rotura no presentan diferencias; por lo tanto, no se ven afectadas estas propiedades del cuero frente a la humedad.

En la fuerza de desgarre (medición en probeta B) se detectan diferencias. A las $24 \mathrm{hs}$ de exposición se detecta el menor valor promedio para la Fuerza. Hasta ese momento la fuerza no está afectada por la humedad. Por lo tanto, en este caso se puede concluir que la propiedad de fuerza se mantiene cuando se lo somete a niveles de humedad hasta las $12 \mathrm{hs}$. 
En las mediciones de la Fuerza con la probeta $\mathrm{C}$ se advierte que no difiere en todos los niveles de humedad a excepción de las 12 hs., donde la fuerza es menor, por lo cual ameritaría una repetición de este ensayo en futuras experimentaciones ya que no hay indicios para explicar el porqué del aumento de la fuerza al pasar de 12hs. a 24hs. de exposición.

Se concluye, además, que es apropiado trabajar con modelos con covariable debido a que las áreas del material difieren significativamente.

Para el tratamiento temperatura no se detectan diferencias significativas en tensión, en alargue a la rotura (probeta A) ni en la Fuerza (probetas B y C). Solo hay diferencias en el alargue porcentual a partir de los 60 grados a partir del cual esta característica presenta menores valores promedio.

Se puede concluir, en general, que las características del cuero analizado son satisfactorias cuando están sometidas a condiciones extremas de humedad, ya que hasta las 12hs las características del cuero no se ven afectadas. En relación a la temperatura, el comportamiento es similar, ya que hasta $\operatorname{los} 40^{\circ} \mathrm{C}$ las condiciones del cuero no se ven afectadas.

Todo esto contribuye significativamente en profundizar las características y el comportamiento del cuero en distintas condiciones de uso. Esta investigación servirá para mejorar las técnicas de almacenamiento y de uso para las distintas prestaciones que cubre el cuero.

\section{REFERENCIAS}

Boccone R.L., Fontana J. A. y Kamp G. (1977a). Distribución de propiedades medibles con elastómero en cueros soft para calzados. Recuperado de: http://catalogo.latu.org.uy/opac_ css/doc_num.php?explnum_id=214.

Boccone R.L., Fontana J. A. y Kamp G. (1977b). Estudio de la relación existente entre las cargas de rotura del cuero medidas con el dinamómetro y el elastómero. Recuperado de: http:// catalogo.latu.org.uy/opac_css/doc_num.php?explnum_id=213.

Boccone R.L. y Fontana J. A. (1979a). Factores que influyen en la resistencia al desgarro de cueros bovinos para Vestimenta. Recuperado de: http://catalogo.latu.org.uy/opac_css/doc_ num.php?explnum_id=216.

Boccone R.L. y Fontana J. A. (1979b). Modificaciones en el curtido de cueros bovinos para vestimenta que mejoran la resistencia al desgarro. Recuperado de: http://catalogo.latu.org. uy/opac_css/doc_num.php?explnum_id=215

IRAM 8509 (2006). Cueros y calzados. Ensayos físicos y mecánicos. Determinación del espesor. ICS: 59.140.30: Buenos Aires.

IRAM 8511 (2006) Cueros y calzados. Ensayos físicos y mecánicos. Determinación de la resistencia a la tracción y del alargamiento porcentual. ICS: 59.140.30: Buenos Aires.

IRAM 8513 (2006). Cueros y calzados. Ensayos físicos y mecánicos. Determinación de la resistencia al desgarramiento mediante el método de dos bordes. ICS: 59.140.30: Buenos Aires. 
IRAM 8514 (2006). Cueros y calzados. Ensayos físicos y mecánicos. Determinación de la resistencia al desgarramiento en un punto de costura. Método de los dos orificios. ICS: 59.140.30: Buenos Aires.

IRAM 8522 (2005). Cueros y calzados. Ensayos químicos, físico-mecánicos, y de solidez del color. Zonas de muestreo. ICS: 59.140.30: Buenos Aires.

IRAM 8555 (2005). Cueros y calzados. Preparación y acondicionamiento de probetas para ensayos físicos y mecánicos. ICS: 59.140.30: Buenos Aires.

Silva Trejos, P. (2005). Propiedades físicas y químicas del cuero para calzado de seguridad. Tecnología en Marcha, 18(1).

Wenge Y. (1999). The mechanical properties of leather in relation to softness (Tesis doctoral). Universidad de Leicester, Leicester. 